EL sindicato marítimo en el éter: audiciones radiales de un gremio en la Argentina de los años '30.

Laura CARUSO

Avances del Cesor, Año XII, V. XII, No 12, Primer semestre 2015, pp. 53-70.

ISSN 1514-3899 / ISSNe 2422-6580 - http://web2.rosario-conicet.gov.ar/ojs/index.php/AvancesCesor/index

\title{
El sindicato marítimo en el éter: audiciones radiales de un gremio en la Argentina de los años ' 30 *
}

\section{The maritime union in the ether: radio shows of a trade union in Argentina during the '30s}

\author{
Laura Caruso \\ Consejo Nacional de Investigaciones Científicas y Técnicas \\ Instituto de Altos Estudios Sociales \\ Universidad Nacional de San Martín \\ Universidad de Buenos Aires \\ (Argentina) \\ lauracaruso@gmail.com
}

\section{Resumen}

Este trabajo analiza la participación del gremio de los trabajadores marítimos en la radiofonía como una experiencia valiosa y singular, en la década de 1930. Al mismo tiempo aborda esa participación en la radiofonía argentina desde una perspectiva que ilumina los usos y las formas de apropiación de ese medio de comunicación por parte del sindicato como herramienta para la construcción de una estrategia gremial propia, en el caso de las conferencias realizadas por la Unión Obrera Marítima de Buenos Aires a fines de esa década en Radio Cultura.

Palabras Clave: Unión Obrera Marítima - Audiciones radiales - Construcción sindical

\begin{abstract}
This paper analyzes the participation of the maritime worker's union on the Argentine's radio in the thirties as a unique and valuable experience. At the same time it addresses such participation from a perspective which sheds some light into the uses and forms of appropriation of the media by the Trade Union as a tool for the construction of a trade strategy, as shown by the case of the conferences conducted by the Unión Obrera Marítima of Buenos Aires at the end of that decade in Radio Cultura.
\end{abstract}

Key Words: Unión Obrera Marítima - Radio shows - Unión development

\footnotetext{
*. Agradezco los comentarios y sugerencias de Ana Lía Rey que enriquecieron esta versión final del trabajo.
} 


\section{Introducción}

Dentro del conjunto de las industrias culturales, la radio fue una de las más significativas de las surgidas en el período de entreguerras. En tanto parte vital de los cambios socioculturales y políticos, el espacio radial participó de procesos fundamentales como la conformación de una identidad nacional, la construcción de públicos entre sus oyentes, y de un campo cultural y comercial formado por diversos géneros. De los distintos grupos sociales y políticos, los sindicatos tuvieron una limitada y tardía participación como actores en el proceso de estructuración y desarrollo de la programación radial. Aunque su intervención, en este sentido, se diera de manera incipiente y acotada, la experiencia del gremio marítimo motoriza el interés en torno al papel de las organizaciones obreras dentro del espacio radial, así como también sobre el papel de la radio como medio para desarrollar su política sindical.

Mientras los trabajos sobre la historia de los medios de comunicación carecen de referencias sobre la participación de las organizaciones obreras, la historiografía sobre el movimiento obrero y la izquierda atiende a ciertos aspectos de la cultura de masas y la cultura obrera, donde el deporte, la educación, la prensa y la literatura tienen un espacio destacado, no así la radio. ${ }^{1}$ Aquí la participación del gremio

1. Sobre los primeros, ver KARUSH, Matthew, Cultura de clase: Radio y cine en la creación de una Argentina dividida (1920-1946), Ariel, Buenos Aires, 2013; BERRADE, Martín, El Mundo, la radio... un recorrido por el esplendor de la BBC argentina, Corregidor, Buenos Aires, 2009; MATALLANA, Andrea, Locos por la radio. Una historia social de la radiofonía en la Argentina, 1923- marítimo, los usos, contenidos y apropiaciones del espacio radial para su construcción sindical particular, es motivo de reflexión y análisis. La inicial intervención del gremio de trabajadores marítimos en la radio, a fines de la década de 1930, puede resultar una experiencia valiosa y singular. Este trabajo indaga así la participación de la Unión Obrera Marítima (UOM) en la radiofonía nacional, a través de una serie de audiciones que su Comisión Directiva realizó en LR 10 Radio Cultura entre 1937 y 1938, considerando tanto la historia del movimiento obrero y la radio en aquel contexto, vinculados a los cambios económicos, sociales, políticos y culturales más amplios.

La idea que guía este trabajo sostiene que, a través de la puesta al aire del conjunto de conferencias radiales, aquel sindicato de los trabajadores marítimos impulsó una estrategia gremial vinculada al Estado, y también de cara hacia sus afiliados y a sus competidores (otros gremios), acción que, más allá de su efectividad y resultado, contribuyó a la consolidación de ciertas formas gremiales y de la relación Estadosindicatos en aquellos años. Aquí se destaca el rol de la radio en la construcción de la política gremial de la UOM, como una experiencia

1947, Prometeo, Buenos Aires, 2006; GALLO, Ricardo, La radio, ese mundo tan sonoro, Corregidor, Buenos Aires, 2001, T. I, “Década del 20 de la radiodifusión”, y T. II: Los años '30; ULANOVSKY, Carlos, Días de radio. Historia de la radio argentina, Espasa Calpe, Buenos Aires, 1996; BOSETTI, Oscar, Radiofonías. Palabras y sonidos de largo alcance, Colihue, Buenos Aires, 1994; SARLO, Beatriz, La imaginación técnica. Sueños modernos de la cultura argentina, Nueva Visión, Buenos Aires, 1992. Dentro del segundo grupo se destacan CAMARERO, Hernán, A la conquista de la clase obrera. Los comunistas y el mundo del trabajo en la Argentina, 1920-1935, Siglo XXI, Buenos Aires, 2007, capítulo 4; PORRINI BERACOCHEA, Rodolfo, Izquierda uruguaya y culturas obreras en el tiempo libre: Montevideo (1920-1950), Tesis doctoral, Universidad de Buenos Aires, 2011 (mimeo). 
valiosa para establecer cruces entre diversos campos historiográficos y reconstruir así una experiencia histórica singular. A partir de este planteo, la radio es considerada un territorio propicio no solamente para intelectuales y aficionados, sino también para los trabajadores y sus organizaciones, considerando a la vez su participación en la radiofonía argentina desde una perspectiva que ilumina los usos y las formas de apropiación de ese medio de comunicación por parte del sindicato como una herramienta para la construcción política.

Junto a intelectuales, escritores, guionistas, actores y actrices, músicos, locutores, presidentes y funcionarios, los trabajadores $\mathrm{y}$ sus organizaciones pueden $\mathrm{y}$ deben ser pensados no únicamente como consumidores del espacio radial, sino como productores, por cierto marginales y tardíos, en la radiofonía argentina. Además de técnicos y operarios, fueron sujetos activos en el desarrollo de ciertos contenidos y usos, y a través de sus organizaciones se apropiaron y habitaron el éter, sumando así a la radio a su amplio repertorio de canales e instrumentos para el desarrollo de su política gremial. En consecuencia, por un lado, esta investigación se pregunta por la presencia de organizaciones obreras en el espacio radial de los años ' 30 , un problema aquí planteado si bien no resuelto, dado su extensión y complejidad. Por otro, se interroga específicamente por el rol de la radio en el desarrollo de la política gremial del período a partir del caso marítimo. La apuesta es alta, y requiere de poder transitar entre diversos abordajes historiográficos: la historia social del mundo del trabajo, la historia de los medios de comunicación, o de manera más amplia, del desarrollo de las industrias culturales masivas, y la historia del período particular de entreguerras.

Las primeras transmisiones radiales en Argentina se dieron en los años de 1920 de la mano de pioneros y aficionados. Recién en la década siguiente la radio tuvo un desarrollo y una importancia social, cultural y política que alcanzó a la sociedad toda, transformándose en un nuevo fenómeno comunicacional y cultural. $\mathrm{Su}$ poder inmaterial, dada la invisibilidad de su función ante la desaparición de los "hilos" presentes en el teléfono y el telégrafo, permitía la comunicación masiva, inmediata y a distancia. ${ }^{2}$ El mensaje radiofónico cobró así un alto grado de eficacia para la comunicación social de gran alcance, con una enorme diversidad de recepciones y audiencias, llegando a los hogares a bajo costo, y produciendo una comunicación empática y afectiva. ${ }^{3}$ La radio se convirtió así en el primer medio que masificó el acceso a determinados contenidos, convirtiéndose, a través de la expansión de emisoras y públicos, en un fenómeno de masas que trasvasó los límites sociales de la burguesía, llegando en la década de 1930 a ser incorporada a los usos cotidianos por un sector amplio y diverso de la sociedad. ${ }^{4}$

Tal desarrollo radial fue parte y producto de cambios profundos en el país, de un verdadero proceso de transformación social. Como se ha sostenido, las consecuencias de la crisis mundial de 1929, el desarrollo de la sustitución de importaciones y otras políticas impulsadas

2. SARLO, Beatriz, "La radio, el cine, la televisión: comunicación a distancia” y "Médicos, curanderos y videntes”, en SARLO, Beatriz, La imaginación técnica..., Op. Cit., p. 111.

3. ULANOVSKY, Carlos, Dias de radio..., Op. Cit.

4. SARLO, Beatriz "La radio, el cine, la televisión...", Op. Cit., pp. 118, 119 y134. 
por los gobiernos conservadores, apuntalaron en diversos sentidos una vertebración más acabada y de alance nacional. ${ }^{5}$ Dichas políticas, si bien buscaban preservar el orden social que pensaban amenazado, resultaron en múltiples y concatenadas transformaciones: la expansión del parque industrial existente y de la oferta, del consumo y del nivel de ocupación, un crecimiento y reordenamiento poblacional caracterizado por la expulsión de población del campo hacia las ciudades, y la consecuente concentración urbana en la región pampeana en general, y en torno a la Ciudad de Buenos Aires en particular. ${ }^{6} \mathrm{~L}$ a radio emergió entonces como un medio de comunicación poderoso, un artefacto característico de esos multiplicados hogares obreros y de clase media. En ella, se podía escuchar música, noticias, radioteatros, así como conocer e imaginar el país entero a través de las transmisiones de turismo carretera, de boxeo o fútbol. La radiofonía colaboró en la formación y construcción de un público y de una identidad nacional, siendo un canal efectivo para fortalecer el proceso de homogenización cultural, por su capacidad de generar un sentimiento de comunidad a distancia y por permitir vivenciar una unidad nacional invisible, imaginaria, haciendo de la idea de Nación vinculada al Estado y la política una experiencia más cercana, emotiva y cotidiana. ${ }^{7}$ La radio irradiaba un

5. BALLENT, Anahi y GORELIK, Adrian, "País urbano o país rural: la modernización territorial y su crisis”, en CATTARUZZA, Alejandro (director), Crisis económica, avance del Estado e incertidumbre politica (1930-1943). Nueva Historia Argentina, T. VII, Sudamericana, Buenos Aires, 2001.

6. KOROL, Juan Carlos, "La economía” en CATTARUZZA, Alejandro (director) Crisis Económica..., Op. Cit.

7.BARBERO, Martín Jesús, De los medios a las mediaciones: comunicación, cultura y hegemonía, Editorial modo de vida y una identidad común, valores y comportamientos, actuando como un mecanismo de integración social que llegaba a todos los rincones del país, consolidando imágenes e ideas de un nosotros nacional. ${ }^{8}$ En tanto fenómeno multidimensional, político, social y cultural, la radio pudo entrar de forma masiva y privilegiada en el espacio doméstico y cotidiano de la población, y en particular, en los hogares proletarios.

\section{Radio, política y Estado}

En su desarrollo, la radio supo recortar campos de audiencia diferenciados y fue capaz de satisfacer las necesidades informativas, recreativas, educacionales y demás de variados sectores sociales. Como sostiene Andrea Matallana, una audiencia masiva se conformó a través de una interrelación entre los gustos del público y la programación. ${ }^{9}$ En los primeros años, surgieron varias emisoras, como Radio Sud América, Radio Brusa (luego Exelsior), Radio Libertad (más tarde Mitre), Radio Nacional (posteriormente Belgrano), Quilmes Broadcasting, Estación Flores, Radio Callao, Radio Olivos y Radio Municipal, la primera emisora oficial del país. Entre estas se destacó Radio Cultura, una pujante actividad comercial y publicitaria dentro del éter y un potente negocio, impulsado por el consumo

Gustavo Gil, Barcelona, 1987, p. 225. Mientras que la escuela y el servicio militar obligatorio fueron pensados como espacios de consolidación de una identidad nacional en décadas pasadas, para 1930 dicha identidad estaba en total construcción y reconfiguración.

8.KARUSH, Matthew, Cultura de clase..., Op. Cit., p. 89.

9. MATALLANA, Andrea, Locos por la radio... Op. Cit., p. 19. 
masivo de una sociedad creciente y compleja. ${ }^{10}$

En el acotado dial de comienzos de 1920, Radio Cultura fue la primera emisora comercial que financió sus espacios con publicidad, mediante flashes y auspicios de programas. Si en sus primeros años esta radio se abocó a conquistar al público femenino desde su flamante locación en la Estación Palermo, tras haber pasado por el Hotel Plaza, pronto se convirtió en una emisora más diversa y pujante. ${ }^{11}$ Por ella pasaron orquestas de tango, cantores y grupos folclóricos, pianistas de jazz y una variedad de teleteatros, en sintonía con programas como "La mujer y el jazz", y las audiciones culinarias. ${ }^{12}$ Pionera y exitosa en los años '20, ante la cada vez más numerosa competencia, Radio Cultura sufrió un gradual corrimiento del centro de la escena radial en la década siguiente, superada por Radio Belgrano y Splendid y Radio El Mundo. ${ }^{13}$

10. ROCCHI, Fernando, "Consumir es un placer: la industria y la expansión de la demanda en Buenos Aires a la vuelta del siglo pasado", en Desarrollo Económico, Buenos Aires, 1998, V. 37, No 148; "Inventando la soberanía del consumidor: publicidad, privacidad y revolución del mercado en la Argentina, 18601940", en DEVOTO Fernando y MADERO Marta (compiladores) Historia de la vida privada en la Argentina, Taurus- Alfaguara, Buenos Aires, 1999.

11. MATALLANA, Andrea, Locos por la radio..., Op. Cit., p. 19. La radio y su función en el hogar también resignificó el lugar de la mujer ama de casa y generó nuevos rubros y actividades para este público específico, como los radioteatros.

12. Esta emisora contaba además con una revista de su mismo nombre, con una tirada de 20.000 ejemplares y distribución gratuita hasta junio de 1923. También se dedicó a la venta de discos y receptores. BOSETTI, Oscar, Radiofonias..., Op. Cit.

13. GALLO, Ricardo, La radio, ese mundo tan sonoro..., Op. Cit., T. II. BERRADE, Martín, El Mundo, la radio..., Op. Cit.
Si bien abandonó el podio de la radiofonía nacional en 1930, Radio Cultura no dejó de ser importante dentro de las 18 emisoras que existían en la Capital Federal. ${ }^{14}$ En 1931 fue comprada por Jaime Yankelevich, uno de los creadores de la estructura radial y comercial en la Argentina, quien asumió diversos desafíos para construir un modelo de industria del entretenimiento, según un interesante trabajo de Andrea Matallana. Allí se muestran los múltiples y complejos vínculos que este empresario tejió en dicho recorrido con diversos gobiernos, llegando a conformar la primera cadena argentina de broadcastings, creando, comprando y asociando diversas emisoras de la Capital y el Interior. ${ }^{15}$ Precisamente esta vinculación con las autoridades estatales mostraba, por un lado, la importancia de la radio en la política y, por otro, un creciente papel del Estado en la expansión y evolución de la industria radial, avanzando de manera lenta pero firme en su injerencia sobre los contenidos y gerenciamiento, más allá del otorgamiento de licencias. Esto se plasmó primero en una incipiente normativa, como el reglamento confeccionado entre 1933 y 1934 que definía licencias, concursos, formas de decir y a quienes contratar. Segundo, en la aparición de emisoras estatales y en los usos del espacio radial por jefes de Estado, como los discursos en cadena

14. ROCCHI, Fernando, "La americanización del consumo: las batallas por el mercado argentino, 19201945”, en BARBERO María Inés y REGALSKY, Andrés (editores) Estados Unidos y América Latina en el siglo XX. Transferencias económicas, tecnológicas y culturales, EDUNTREF, Buenos Aires, 2003.

15. MATALLANA, Andrea, "Inventando la radio comercial: apuntes para una biografía de Jaime Yankelevich", en Revista de Instituciones, Ideas y Mercados, Mayo 2013, № 58 pp. 147-166. Para los años previos a 1940 no se menciona información que permita dimensionar el vínculo de Yankelevich con las autoridades del Poder Ejecutivo Nacional. 
nacional del primer presidente de facto José $\mathrm{F}$. Uriburu, o las campañas electorales de Agustín P. Justo (1932) y Roberto M. Ortiz (1938) por Radio Belgrano. ${ }^{16}$ Así, la radio también fue pieza clave en la consolidación de proyectos y liderazgos políticos, como en el caso de Juan D. Perón. ${ }^{17}$

En la década de 1930, el Estado también tuvo una mayor intervención en los conflictos obreros y en la estructura gremial en plena transformación. En una coyuntura de mejora económica y cierta atenuación de la represión política de los gobiernos de Uriburu y Justo, los trabajadores desarrollaron acciones ofensivas en función de nuevas y consolidadas formas de organización de carácter nacional y con una política de acercamiento al poder estatal. Los sindicatos por rama o actividad presentes en todo el país se tornaron el modelo deseable y posible de organización obrera, un modelo sindical centralista y fuerte, atravesado a su vez por tensiones internas e impugnaciones externas. La construcción de tales sindicatos y su consolidación fue de la mano de su vinculación con el Estado. ${ }^{18} \mathrm{Si}$ bien el caso emblemático fue la Unión Ferroviaria (UF), los gremios marítimos no estuvieron ajenos a este proceso. ${ }^{19}$

16. GALLO, Ricardo, La radio, ese mundo tan sonoro..., Op. Cit., T. II.

17. TORRE, Juan Carlos y PASTORIZA, Elisa, "La democratización..., Op. Cit.

18. HOROWITZ, Joel, "El movimiento obrero", en CATARUZZA, Alejandro (director) Nueva Historia..., Op. Cit.

19. Sobre el modelo sindical de la UF, ver HOROWITZ, Joel, "Ideologías sindicales y políticas estatales, 19301943", en Desarrollo Económico, Buenos Aires, 1984, V. 24, No 94, pp. 275-296; HOROWITZ, Joel, "Los trabajadores ferroviarios en la Argentina (1920-1943). La formación de una elite obrera" en Desarrollo Económico,

\section{Historia de un gremio}

Adelantados en la tarea de construcción de un gremio de alcance nacional, los trabajadores marítimos ya en 1910 habían formado la Federación Obrera Marítima (FOM). Dicha organización materializó la unidad de todas las categorías de trabajadores embarcados de las secciones a bordo y de las filiales territoriales en los puertos del interior, al agrupar a marineros, foguistas, mozos y otros trabajadores embarcados de sala de máquinas, cubierta y cocina, de todo el país. La FOM fue así uno de los primeros sindicatos por actividad de carácter nacional, una organización clave en las primeras décadas del siglo XX y pilar de la Federación Obrera Regional Argentina del noveno congreso (FORA IX). Hasta los años '20 la dirección política de ambas centrales fue protagonizada por militantes del sindicalismo revolucionario vernáculo, que pronto de convertiría en sindicalismo a secas. ${ }^{20}$ Sin embargo, a mediados de los años '20 surgió otro sindicato en el sector marítimo que compitió por la representación de los obreros embarcados. La UOM, creada el 23 de diciembre de 1925, persistió como gremio marítimo de empresa hasta 1946, cuando se inició el proceso de organización de un sindicato único marítimo, que la unió finalmente a la FOM y a muchos otros centros de oficiales, capitanes y maquinistas, durante el primer gobierno peronista.

Ahora bien, el origen de la UOM es y fue

Buenos Aires, 1984, V. 25, No 99, pp. 421-446.

20. DEL CAMPO, Hugo, Sindicalismo y peronismo. Los comienzos de un vinculo perdurable, Siglo XXI, Buenos Aires, 2005. 
motivo de polémica. Ocurrió en una coyuntura muycompleja paraelgremialismo marítimo,tras un periodo de reflujo a comienzos de la década de 1920. Fue sospechada de haber contado con respaldo estatal y empresario, y conocida como un gremio propatronal impulsado por la compañía naviera Mihanovich, la mayor empresa de navegación de cabotaje del país. Años después, la UOM llegó a contar con una participación más extensa y una militancia de base de los trabajadores del sector y militantes de diversas corrientes obreras, como el socialismo y el comunismo, opuestos a la conducción sindicalista, pragmática y autodefinida apolítica de la FOM, gremio con el cual competiría la UOM por casi dos décadas.

La carencia de trabajos acerca de la UOM sólo puede ser aplacada por la reconstrucción histórica a partir de datos fragmentarios y dispersos. Luego de la huelga parcial marítima impulsada en 1920 por la FOM en defensa del control sindical a bordo contra la política de Mihanovich, se abrió una coyuntura de debilidad y represión para el gremio marítimo y para al movimiento obrero en general. La FOM entró en un proceso interno complejo, regresivo y pendular, y tras la derrotada huelga en contra de la Ley de Jubilaciones impulsada por el gobierno de Marcelo T. Alvear en 1924, se creó la UOM. ${ }^{21}$ Dicha ley, que contemplaba altos aportes de los trabajadores, fue apoyada

21. Sobre la huelga contra el proyecto de ley jubilatoria, ver HOROWITZ, Joel, "Cuando las elites y los trabajadores coincidieron: La resistencia al programa de bienestar patrocinado por el gobierno argentino, 19231924", en Anuario IEHS, Tandil, 2001, № 16; AQUINO, Cristian, "Izquierda y movimiento obrero: Estrategias y discursos del sindicalismo revolucionario a propósito de la ley de jubilaciones de 1924", ponencia presentada en XI Jornadas Interescuelas / Deptos. de Historia, Tucumán, septiembre de 2007. por los centros de oficiales, resurgiendo así una diferencia dentro de las organizaciones del mundo gremial marítimo, que reinstalaba la dificultad de una unidad de criterio y de acción, una unidad política que la FOM y los centros de oficiales había construido en el período inmediatamente anterior. ${ }^{22} \mathrm{La}$ ruptura de la solidaridad con los gremios de capitanes y oficiales, sumada a la desarticulación de la FOM y su pérdida del control del ingreso y de las condiciones de trabajo a bordo definieron la coyuntura para el surgimiento de otros gremios dentro del sector. Surgió así la UOM, con características bien particulares, diferenciada de la FOM no sólo por su política, sino también en su forma de organización, ya que se estructuró como un sindicato de empresa, que agrupaba a trabajadores y oficiales de la compañía Mihanovich. ${ }^{23}$

Sospechada de amarillismo por contemporáneos e historiadores, la UOM contó con el apoyo programático e institucional de los oficiales y sus organizaciones -como el Centro de Capitanes y Oficiales de Ultramar y la Unión Naval de Oficiales- y con el sustento estatal a través de las gestiones del Prefecto General de Puertos Ricardo Hermelo. ${ }^{24}$ También sumó el apoyo del gremio más fuerte

22. CARUSO, Laura, "Control a bordo: la Federación Obrera Marítima, 1916-1921”, en DICÓSIMO, Daniel y SIMONASSI, Silvia (compiladores) Trabajadores y empresarios en la Argentina del siglo XX: indagaciones desde la historia social, Prohistoria Ediciones, Rosario, 2011, pp. 21-34.

23. El predominio que tuvo Mihanovich en el transporte fluvial y marítimo se mantuvo durante décadas, a pesar del cambio de propietarios que en 1930, puso al frente de la empresa al grupo familiar Dodero, que había ejercido la gerencia de la compañía por años antes de comprarla.

24. Hermelo sería el Jefe de Policía tras el golpe de estado de septiembre de 1930 . 
y con mayor presencia política de entonces, la UF. Un estudio realizado por la Confederación General del Trabajo (CGT), a pedido de la FOM, afirmaba categóricamente que "la UOM es una organización patronal", una "oficina de embarque de la compañía". ${ }^{25}$ Más allá de esta caracterización, lo cierto es que el sindicato surgió de la Junta Reorganizadora del Gremio Marítimo, formada tras la derrotada huelga de 1924, donde oficiales y patrones tuvieron una fuerte presencia. Esta entidad tuvo respaldo estatal y patronal para gestionar la conformación de las tripulaciones, y para representar a los trabajadores del sector como interlocutor válido ante empresas y organismos estatales, aunque marineros y demás tripulantes continuaran mayoritariamente agremiados en la FOM. Con el tiempo, como se ha señalado, una creciente militancia sindical -crítica de la burocratización de la FOM- dotó de nuevo contenido a la UOM, protagonizada por militantes comunistas y socialistas. ${ }^{26}$ Los intentos de unidad entre ambos gremios fueron recurrentes, siendo uno de los impulsos más fuertes en este sentido el gestionado por el secretario general de la FOM, Fortunato Marinelli, ${ }^{27}$ muy criticado por otros dirigentes

25. CGT, periódico de la Confederación General del Trabajo, 1/11/1935, No 81, pg. 3, "Un poco de historia de las organizaciones marítimas. Por ella se concluye que la UOM es una organización patronal”.

26. DI TELLA, Torcuato, Perón y los sindicatos. El inicio de una relación conflictiva, Ariel, Buenos Aires, 2003.

27. Fortunato Marinelli (1897-1945) comenzó su extensa militancia sindical en 1913 entre los Constructores de Carruajes. Fue secretario del Sindicato de Artes graficas y miembro de la Agrupación de Pintores Sindicalistas. Tras su paso por la FOF, en 1919 ingreso a la FOM con una importante participación y actividad. Fue su secretario general entre 1933 y 1945, además de miembro de la redacción de los periódicos La Argentina, La Acción y La Libertad (1927- 1934). En 1942 paso a ser director de la Caja de Jubilaciones, y fue delegado ante la OIT en 1938. del llamado sindicalismo revolucionario, como Sebastián Marotta. ${ }^{28}$

Hacia fines de la década de 1930, momento en que salieron al aire las conferencias, la UOM contaba con aproximadamente 4.200 socios. ${ }^{29}$ Tenía una secretaría y una bolsa de trabajo, a causa de la alta desocupación sufrida en el sector en años anteriores, que funcionaba como oficina de trabajo con relativo éxito, incluso en épocas de menor embarque, por fuera de la estación de cosecha y exportación. Apenas inaugurada, a mediados de 1938, la Bolsa de Trabajo logró colocar tripulantes en las diversas secciones de a bordo, consiguiendo ubicar en los meses de agosto a octubre a 350 trabajadores en promedio, entre los que había contramaestres, timoneles, marineros y serenos, foguistas, caldereros y carboneros, mozos, cocineros y peones. Contaba con una Comisión de Prensa encargada de la edición del periódico El Marino con un mimeógrafo propio. Frente a los reiterados problemas financieros, esta comisión decidió incluir avisos comerciales a comienzos de 1938. Otra comisión muy activa fue la de Biblioteca, que encaró la compra sistemática de libros, la confección de listas de suscripciones y la organización de un espacio propio en la sede gremial. También durante 1938 la Comisión Directiva otorgó selectivamente subsidios a socios enfermos y a familiares de tripulantes fallecidos, a la espera de conseguir la personería jurídica para concretar una política

28. Marotta (1888-1970), uno de los más destacados dirigentes sindicalistas, provenía del gremio de la construcción. Se desempeñó también en el sector grafico, y fue integrante en puestos directivos de la UGT, la CORA, la FORA IX y la USA.

29. El Marino o 7, 11/1938, p. 13. 
integral de subsidios. ${ }^{30} \mathrm{Su}$ proximidad con el Estado se manifestó de diversas formas; casi veinte años después de su creación, en 1943, su acercamiento a la Secretaria de Trabajo y Previsión y a la construcción política impulsada por Juan D. Perón la colocó como gremio alineado. Este hecho profundizó sus diferencias con la FOM, y, sin embargo, tras diversos intentos de unificación, en febrero de 1947 finalmente la UOM, la FOM y los centros de oficiales, maquinistas y otros constituyeron la Confederación General de Gremios Marítimos y Afines (CGGMA) que devendría, por procesos propios del período peronista, en el Sindicato de Obreros Marítimos Unidos (SOMU). ${ }^{31}$

Algunos otros indicios de la historia de la UOM apuntan a su vinculación con grupos nacionalistas, tal como lo destacó Mariela Rubinzal. El nacionalismo obrerista de los años '30, así denominado por la autora, tuvo una relación si no cercana, al menos de admiración y simpatía con la UOM, en su lucha anticomunista, considerando a los miembros de la Unión como "argentinos conocedores de sus necesidades”. ${ }^{32}$ Además de este vínculo político entre organizaciones nacionalistas y el gremio marítimo en cuestión, también Radio Cultura, junto otras emisoras, tuvo un vínculo estrecho con tales organizaciones nacionalistas, al dar cabida a la difusión de

30. El Marino No 7, 11/1938, p. 14.

31. CONTRERAS, Gustavo, Contra Viento y marea. Trabajadores maritimos, sindicalismo y peronismo. Un estudio de la "gran" huelga de 1950, Tesis de Licenciatura, Universidad Nacional de Mar del Plata, 2012 (mimeo).

32. RUBINZAL, Mariela Alejandra, "Los conflictos obreros en la prensa nacionalista: itinerarios de un acercamiento ambiguo al mundo del trabajo (19351943)", en Revista Papeles de Trabajo IDAES-UNSAM, Buenos Aires, 2008, No 3. sus ideas y algunas de sus actividades. Un caso estudiado por Rubinzal fue el de la Comisión Popular Argentina contra el Comunismo (C-PACC), que en 1932 promocionaba y difundía sus acciones en las radios Cultura, La Nación y Nacional. Así ocurrió con la movilización y acto público del 20 de agosto convocados en Plaza Congreso para presentar un petitorio para el exterminio de todo tipo de expresión comunista en el país. ${ }^{33}$

\section{Un sindicato navegando el éter}

En la programación del espacio radial argentino la existencia de emisiones especiales fue una constante. Muchas serían luego incorporadas a la grilla permanente de las emisoras. Dentro de la amplia gama de posibilidades, cobertura de eventos y otros más esporádicos programas, existieron audiciones destinadas a un público particular: los trabajadores del sector marítimo y sus familias. A fines de 1937, comenzaron así las conferencias de la Comisión Directiva de la UOM en el aire de Radio Cultura, en general los días miércoles a las 21 horas.

Como se ha mencionado, para entonces Radio Cultura estaba fuera del podio de las emisoras radiales; tal vez esa condición posibilitó el acceso a ese espacio del gremio marítimo. Sus conferencias estaban dedicadas a un segmento particular de oyentes, siendo secundarias dentro de una programación radial que incluía a figuras como la actriz Olinda Bozan, el actor Roberto Escalada, o el músico, compositor y cantor de tangos

33. RUBINZAL, Mariela Alejandra, "Manifestaciones nacionalistas y católicas en las calles de Buenos Aires (1930-1945)", en Boletín PolHis, Buenos Aires, primer semestre 2012, Año 5, No 9, pp. 191-203. 
Agustín Magaldi, de una llegada a un público amplio y masivo. Entre el paisaje homogéneo que presentaban los contenidos radiales de las emisoras más exitosas en el horario central, donde imperaban los radioteatros y la música como el tango, el jazz y, en menor medida, la música clásica, intercalados con noticias, eventos deportivos y sketches, se desarrolló una heterogeneidad en los bordes del dial y del día, en áreas y horarios marginales. ${ }^{34}$ En esos márgenes tuvo lugar la serie de audiciones pensadas desde una organización gremial de a bordo. La UOM puso al aire nueve audiciones entre diciembre de 1937 y noviembre de 1938, una coyuntura que, en contraste con el lustro anterior atravesado por la crisis y la depresión económica, la alta desocupación, la represión política y la escaza movilización obrera, mostraba nuevas posibilidades y escenarios a nivel económico y político. El avance de la industrialización como salida a la crisis, el consiguiente mayor nivel de empleo, con una nueva composición entre los trabajadores y una mayor presencia de la mano de obra industrial, así como el intenso proceso de organización sindical a nivel nacional de las diversas ramas industriales, y sobre todo, la búsqueda estratégica de un acercamiento con el poder estatal por una parte mayoritaria de esos gremios, definieron un nuevo período de acción obrera. ${ }^{35}$ Los canales para este acercamiento fueron diversos y erráticos, más consolidados en los ámbitos de acción de las instituciones laborales. ${ }^{36} \mathrm{~A}$ diferencia de los años anteriores,

34. MATALLANA, Andrea, Locos por la radio..., Op. Cit.

35. HOROWITZ, Joel, “El movimiento obrero”..., Op. Cit.

36. SURIANO, Juan y LOBATO, Mirta (compiladores) La sociedad del trabajo. Las instituciones laborales en Argentina, 1907-1955, Edhasa, Buenos Aires, 2014; los conflictos gremiales tuvieron resultados en general favorables, y contaron con la activa mediación estatal, a través de su agencia especializada, el Departamento Nacional del Trabajo.

Aquellas conferencias son accesibles a nuestro conocimiento gracias a su publicación y transcripción en las páginas del periódico oficial de la UOM, El Marino. Encaradas como un emprendimiento de propaganda gremial, las audiciones radiales del gremio fueron concebidas por este como una actividad nueva y primordial, dentro de una amplia tarea de mejoramiento del gremio y sus afiliados, que incluía reuniones con autoridades estatales, gestiones para la sanción de leyes laborales como la de jubilación, entre otras soluciones posibles a las "aspiraciones marítimas".

Durante aquel último mes de 1937 y los meses del año siguiente en cada edición del periódico se narraban cada una de las conferencias, apuntando quienes las habían conducido y cuáles fueron las ideas centrales allí volcadas. El paso de una conferencia al soporte papel en aquellos años era habitual en diversos sectores, incluidos la iglesia o autoridades provinciales como el gobernador bonaerense Manuel Fresco. Sin embargo, sin ser una novedad, la transcripción cuasi literal de estas audiciones en un periódico gremial al menos llama a una reflexión sobre

GAUDIO, Ricardo y PILONE, Jorge, "El desarrollo de la negociación colectiva durante la etapa de modernización industrial en la Argentina, 1935- 1943" en Desarrollo Económico, Buenos Aires, julio-septiembre 1983, V. 23, No 90; GAUDIO, Ricardo y PILONE, Jorge, "Estado y relaciones laborales en el período previo al surgimiento del peronismo, 1935-1943" en Desarrollo Económico, Buenos Aires, julio-septiembre 1984, V. 24, No 94. 
la búsqueda de perdurabilidad del discurso ante la utilización de un nuevo medio como la radio, cuya inmaterialidad podría incluso generar incertidumbres y desconfianzas. Lo seguro y conocido, la palabra escrita frente a la palabra intangible que se escucha, pareciera reforzar la necesidad de dejar registro de aquel mensaje esencial por temor a su fugacidad y su pérdida en el tiempo y el espacio. Bajo el título "Nuestras audiciones radiales" aparecen comentadas las conferencias; las dos primeras se compilaron en una única nota publicada en diciembre de 1937. En abril, se reprodujeron los contenidos de las audiciones tres a siete. Una última referencia aparece en mayo de 1938, con las dos últimas "disertaciones para la gente de mar”. Así, periódico y conferencias conforman, durante el tiempo que conviven, un conjunto funcional destinado a la propaganda y la construcción gremial. Tanto en su formato como en sus contenidos, las audiciones radiales parecían la continuación hablada de la prensa gremial escrita. Como mencionamos, la palabra dicha volvía en forma de nota y era transcripta en el papel. Esto expresa, de alguna manera, una estrecha vinculación y retroalimentación entre el nuevo medio de comunicación, la radio, $\mathrm{y}$ aquellos que la precedieron, de los cuales tomó elementos diversos, tópicos, formas y discursos. Es posible pensar entonces que estas conferencias forman parte de un género, una forma de decir y comunicar ya conocida por el gremio, que va transitando diversos soportes. El género conferencia generó así una trama de herramientas y soportes comunicacionales, que incluyó la prensa, las conferencias radiales, y también formas de sociabilidad gremial como las veladas y conferencias -, generando un circuito con similares contenidos que variaba en sus formatos.
En la creación de este circuito, los trabajadores fueron a la vez parte del proceso de producción radial como del conjunto de consumidores de su programación. Como en otros casos, la producción del mensaje radial del gremio marítimo se constituyó a través de transferencias y préstamos de elementos de los medios conocidos, generando en principio un formato y un contenido similar al ya practicado en la prensa y en la sociabilidad, constitutivos de un campo sociocultural común. En el caso sindical marítimo, la conferencia dio forma a un nuevo espacio de comunicación entre el gremio y sus trabajadores. Otro espacio bien tradicional era el periódico gremial. Este, a partir de abril de 1938, incorporó en forma creciente, con mayor asiduidad y tamaño, diversas publicidades de productos y servicios varios (bebidas, cigarrillos, sorteos, empresas de pintura de vapores, despensas y proveedurías marítimas, farmacias y sanatorios, panaderías, confiterías y fondas de la zona portuaria, abogados y otros profesionales). De la misma manera, es posible pensar que en las audiciones radiales también existieron auspiciantes y avisos publicitarios de diverso tipo, y que incluso la publicidad del periódico serviría para comprar el espacio radial. ${ }^{37}$

Las conferencias gremiales no tuvieron un conductor; fueron presididas por distintos miembros del Consejo Directivo de la UOM, como su dirigente máximo, José López, quien debía estar en la audición de inicio del ciclo, pero por motivos laborales recién estuvo en la segunda emisión, el miércoles 8 de diciembre de 1937. La primera conferencia fue conducida entonces por el secretario de la

37. En general, parte de lo recaudado por los avisos del periódico era destinado a un fondo mutual para los asociados. El Marino No 4, 05/1938, p. 7. 
UOM, Daniel Alvarado, también presente en la tercera, quinta y las dos últimas, junto a otros dirigentes, como R. Conde y A. Gutiérrez, quienes también presidieron otras audiciones. La continuidad de las conferencias, si bien espaciada y asistemática, fue suspendida en mayo de 1938, ya que la UOM cedió el espacio radial al Círculo de Oficiales de la Marina Mercante para publicitar e impulsar la sanción de la ley de jubilaciones para la gente de mar.

\section{¿Qué y para quién? Contenidos y destinatarios de las emisiones gremiales}

El Consejo Directivo de la UOM definió al sentido de estas transmisiones: eran fundamentales para la obra y propaganda de sus demandas y necesidades, entre las que se destacaban los reclamos por aumento salarial, la sanción de una ley de jubilaciones y pensiones marítimas, la denuncia de la carestía de vida, la desocupación, el cumplimiento del escalafón y la estabilidad laboral. También se informaba sobre las gestiones realizadas por el sindicato ante las autoridades estatales y patronales. Dicho en otros términos, en estas conversaciones radiales se pretendía comunicar las necesidades de los trabajadores y empleados marítimos, así entendidas por la UOM, así como dar difusión de los medios que traerían una solución y las actividades desplegadas por la organización. Este era el propósito declamado. Entonces, los interlocutores primeros de estas emisiones eran los afiliados al sindicato y los trabajadores de la navegación de cabotaje en general. Sin embargo, aunque los tripulantes fueron los destinatarios primeros y fundamentales del emprendimiento radial de la UOM, no fueron los únicos. Junto a los interlocutores obvios - afiliados o posibles afiliados del sector, sus familias y su entorno - existieron otros de gran importancia en el discurso y la estrategia política de la UOM: las autoridades gubernamentales y la extensa comunidad marítima, aquellos trabajadores de a bordo pertenecientes a otros gremios o carentes de organización. Ambos fueron interpelados desde el espacio radial como parte central de la política sindical de esta organización particular, inmersa en un proceso generalizado de la apelación de los gremios al Estado en el período.

Las conferencias buscaron la atención y la respuesta de las autoridades gubernamentales $\mathrm{y}$ diversas dependencias con las que el sindicato venía construyendo una relación fluida. En la primera audición se explicitaba como propósito del ciclo "hacer conocer a las autoridades y al público en general, algunas de las aspiraciones y problemas que nos afectan a todos los marítimos y que estimamos de improrrogable resolución". ${ }^{38}$ Dicha tarea era fundamental para la política gremial, en tanto el conocimiento público, y en especial por parte del gobierno, era entendido como el primer y efectivo paso en el camino de la concreción de sus demandas. Sostenía así que

los que aspiran a bancas en el Senado y Cámara de Diputados nacionales, demuestran, en general, un desconocimiento absoluto de nuestros anhelos; lo cual significa que si nosotros los interesados no logramos demostrar nuestras necesidades siempre permanecemos huérfanos del apoyo de los poderes públicos. ${ }^{39}$

38. El Marino o 1,12/1937, p. 3, "Las Conferencias Radiales de la Unión Obrera Marítima”.

39. El Marino No 4, 05/938, p. 13 , "Conferencias radiales". 90 Audición. 
La pérdida de esa orfandad, es decir, la búsqueda de protección y apoyo por parte de los poderes públicos, permitiría el avance del gremio, y con él, el mejoramiento de la situación de sus trabajadores, según el discurso gremial. Se interpelaba así a ministros, prefectos, legisladores y otras autoridades gubernamentales, reclamando su activa intervención en el mundo laboral marítimo, y su mediación favorable ante las empresas y ante la competencia gremial. Con esta acción la UOM dotaba al Estado de una potestad no sólo en las cuestiones laborales, sino incluso en la disputa intersindical, en su batalla frente a la FOM, para defender a los "verdaderos marítimos", los propios. La apelación al Estado y la demanda de un diálogo posible y deseable entre éste y el gremio formaban parte, por cierto, del proceso más general desarrollarlo en el seno del movimiento obrero en esa década, antes mencionado. Sin embargo, en la experiencia particular de los trabajadores marítimos, dicho acercamiento databa de un período previo, en un largo y tensionado camino. ${ }^{40} \mathrm{Si}$ bien en los años anteriores tal vínculo con el Estado fue primordialmente impulsado por la FOM, la relación Estadogremios y la intervención estatal formaban parte de una experiencia histórica común del colectivo laboral marítimo, lo cual permite pensar la fortaleza de tales argumentos en sus nuevas y diversas organizaciones. La radio, pero también la prensa y demás canales, servían al gremio para interpelar al poder estatal y sus representantes. La radio se sumaba como medio para el esfuerzo realizado por la UOM de conseguir apoyo e intervención estatal en

40. CARUSO, Laura, Los trabajadores maritimos del Puerto de Buenos Aires: condiciones laborales, organización sindical y cultura politica, 1890-1920, Tesis Doctoral, Universidad de Buenos Aires, 2012 (mimeo), capítulo 6. diversas cuestiones caras a la organización y sus trabajadores (la competencia con los ferrocarriles, la desocupación creciente por los continuos amarres, etc.) Así, el espacio radial fue un nuevo canal dentro de la estrategia de acercamiento al Estado. ${ }^{41}$.

Es necesario que nos dispongamos a hacerles llegar, una vez más, nuestro anhelo de que en el presente año, los gobernantes de nuestro país se ocupen de nosotros sancionando leyes de amparo para los empleados y obreros marítimos, y dándole también, a la marina de cabotaje el apoyo que merece sobre todo franquicias fiscales, combustibles baratos y liberándola de competencia ruidosa. ${ }^{42}$

Desde una posición negociadora y pragmática, tales apelaciones muestran también una clara confianza depositada en el vínculo con el Estado y sus poderes como método válido y eficaz para lograr satisfacer sus reclamos y aspiraciones. Con relación a una serie de entrevistas sostenidas con representante del gobierno, el gremio declaraba:

Íntimamente satisfechos, debemos manifestar que hallamos en el señor Ministro de Marina al gobernante profundamente compenetrado de los problemas que afectan al gremio marítimo, deseoso de encarar su pronta resolución y, sobre todo, dispuesto a prestar su valiosa mediación para que nos sea concedido un aumento razonable... Vaya, pues, por medio de estas palabras, el

41. Por ejemplo, allí se informaban y se solicitaban audiencias con los Ministros, como ocurrió con el de Marina y Obras Públicas, con quienes el $1^{\circ}$ de diciembre de 1937 se reunieron por más de una hora. El Marino No 1, 12/1937, p 1 .

42. El Marino No 3, 04/1938, p. 7, "Nuestras audiciones radiales". 50 Audición. 
agradecimiento de la Comisión Directiva Central de la Unión Obrera Marítima y de los asociados a esta, hacia el alto jefe de la armada que con tanta deferencia atendió a los delegados de esta sociedad. ${ }^{43}$

La interpelación de la UOM al poder público explicita el carácter definidamente conciliador y apelativo, característico de una táctica negociadora y dialoguista, tanto con el gobierno como con la patronal. Tal posición dejaba manifiestamente de lado toda acción directa. La huelga y la movilización como método de acción sindical eran denunciadas y consideradas injustificables:

De los resultados de este último método ya tenemos duras experiencias los obreros marítimos, y podemos decir a los oyentes que sólo nos ha reportado miseria, años de inactividad, luchas estériles, rencores y desconfianzas del capital para implantar nuevas líneas de navegación y, como consecuencia de esta desconfianza, mayor cantidad de desocupados en el gremio.

La necesidad y legitimidad de la intervención estatal excedía al mundo sindical y lo estrictamente laboral. En las audiciones se bogaba por una acción del Estado dentro de la economía nacional en general, y del sector del transporte marítimo en particular, así como también en la organización y desarrollo de la marina de cabotaje y de ultramar, tareas que no podían dejarse libradas al esfuerzo privado. ${ }^{44}$ La potestad de la intervención estatal reclamada por la UOM en las audiciones de la noche llegaba incluso al interior del propio conflicto gremial por la representación de los

43. El Marino № 1, 12/1937, p. 3, "Las Conferencias Radiales de la Unión Obrera Marítima”.

44. El Marino No 3, 04/1938, p. 6, "Nuestras audiciones radiales". 5० Audición.

66 trabajadores:

si se desea que el gremio permanezca en paz, si se desea asegurar la tranquilidad social del país, si se quiere evitar perturbaciones fomentadas por los eternos negociadores que saben explotar la ingenuidad de las masas obreras y sembrar el descontento agitando sus necesidades más sentidas, para arrastrarlas a luchas estériles muy desorbitadas, es necesario, es imprescindible, que los señores gobernantes se dispongan a remediar el mal a tiempo. ${ }^{45}$

El contenido de estas audiciones, su mensaje, demandas, argumentos y expectativas, no parece novedoso ni se diferencia de las posturas clásicas del sindicalismo en general de los años '30. La táctica de la negociación, el discurso de una armonía de clases y el acercamiento a los poderes del Estado en diversas instancias eran entonces característicos de buena parte de los gremios, y por cierto, de la UOM. Lo que se destaca aquí es la utilización y apropiación del espacio radial como medio para la construcción de tal política gremial, y lo fue claramente en el caso del sector marítimo.

Ciertas demandas sostenidas por la UOM frente al Estado pueden ser incluso asimiladas al discurso patronal, por ejemplo la competencia desleal de los ferrocarriles y el perjuicio para el negocio naviero y la actividad del sector, las que ocuparon un espacio importante en las conferencias. El problema de las tarifas diferenciales permitidas por el gobierno a favor del ferrocarril venía siendo denunciado por las navieras desde principios de siglo. Las mismas perjudicaban el tráfico

45. El Marino No 1,12/1937, p. 7, "Nuestras audiciones radiales", 7º Audición. 
comercial de cabotaje, junto a preferencias impositivas y el menor precio del combustible. Estos "inconvenientes patronales" tenían una repercusión directa en la lucha gremial, ya que era el argumento utilizado por las empresas navieras para negarse a conceder los aumentos salariales reclamados, como sucedió en octubre de 1937, cuando la UOM presentó un petitorio demandando un aumento y el pago de horas extras. La respuesta patronal argumentó la ruina que causaba el precio del combustible y la competencia del transporte ferroviario, que imposibilitaba cualquier aumento. ${ }^{46}$ Sin dudar de la buena voluntad de estos empresarios, la UOM llamaba a hacer suya esta causa que repercutía directamente en el bienestar obrero, en el nivel de ocupación y en los salarios.

Por último, es posible encontrar un tercer destinatario de las conferencias: la comunidad laboral marítima. La UOM interpelaba de múltiples maneras, a través del periódico, de actividades, y también radialmente, a los trabajadores marítimos de las diversas secciones, en función de su lucha política con la FOM, con la que disputaba la legitimidad de la representación de esos trabajadores ante sí mismos, ante las empresas y el Estado. El debate era explícito:

Es posible que alguien con criterio simplista pueda decirnos ¿pero que puede interesarles a los obreros marítimos las tasas impositivas, competencia y demás trabas que soporta la marina mercante?... Eso diría alguno de los que todo lo resuelven en forma unilateral, puede interesarles a los armadores y a ellos les toca buscar la solución... lo que deben hacer los obreros, -exclamarían los lideres de ciertas tituladas organizaciones obreras revolucionarias- es exigir más sueldo,

46. El Marino № 1, 12/1937, p. 1. menos horas de trabajo, y en ultimo termino deben tender a apropiarse del contralor de los buques para explotarlos en beneficio de sus tripulantes.

Desde el espacio radial, a la vez que dialogaba y apelaba con el gobierno y las empresas, la UOM debatía y competía con otras organizaciones y líneas de construcción gremial. Lo hacía apelando al interés común, al de la Nación y de la "gran familia marítima”, una idea de comunidad armónica y conciliable, basamento de un discurso integrador y de armonía de clases. Por sobre la heterogeneidad étnico-regional y la fuerte diferenciación jerárquica entre tripulantes y oficiales, característica del mundo laboral marítimo plagado de tensiones y conflictos, la construcción de esta idea de comunidad con vínculos tan cercanos y de familiaridad no era nueva, pero tal vez si su eficacia. ${ }^{47}$ Los reiterados conflictos a bordo entre oficiales y tripulantes no lograban desafiar la fuerte noción de unidad afectiva, necesaria para la supervivencia misma en viaje. Las diversas organizaciones sindicales procesaron tales conflictos de diferentes formas y con diversos sentidos; en principio, reforzando la noción de disciplina, emparentada con las jerarquías entre categorías y secciones. Pero también lo hicieron consolidando la idea de familia marítima frente a una realidad compleja y conflictiva. Esta imagen familiar permitía consolidar fuertes consensos entre oficiales y tripulantes, en torno a la necesidad de una organización laboral jerárquica y disciplinada,

47. Sobre la organización laboral marítima ver CARUSO, Laura, "Onde manda capitão, não governa marinheiro"? O trabalho marítimo no rio da Prata, 1890-1920", en Revista Mundos do Trabalho, publicación semestral del Grupo de Trabajo "Mundos do Trabalho", ANPUH, V. 3, SP, 2010. 
no cuestionada ni por los gremios ni por el conjunto de los actores del sector. La construcción identitaria de la familia marítima confrontaba con las características laborales del sector, pero al mismo tiempo las dotaba de un nuevo marco de sentido. La familia proyectaba una imagen inclusiva, carente de divisiones y conflictos, con un orden interno necesario e incuestionable, que permitía representar a ese colectivo laboral de una manera armónica. Así, quitaba visibilidad a las tensiones reales surgidas de la jerarquía y la fragmentación del trabajo marítimo, aspectos que organizaban muchos, sino todos, los aspectos de esa vida obrera. En la década de 1910, la "familia fomista" se había caracterizado por la acción directa y el enfrentamiento asiduo con una poderosa patronal. ${ }^{48}$ En los años '30, la UOM sostuvo una idea de familia aún más amplia, donde empresas y Estado convivían con el gremio y sus trabajadores. Si bien ambas ideas de familia marítima se construyeron en oposición a aquella realidad compleja y fragmentaria de la vida a bordo, la "familia marítima uomista" en su amplitud y conformación era peculiar. Ciertos marítimos no formaban parte de ella, además de los crumiros, esquiroles o rompehuelgas de antaño; eran los trabajadores de la FOM. Quienes quedaban fuera de la comunidad eran los falsos marítimos, "que no conocen siquiera el planchado por donde se sube a bordo". ${ }^{49} \mathrm{El}$ medio radial servía para unir a la dirección gremial y a esa familia marítima, como un primer círculo de oyentes, para consolidarla y buscar su perdurabilidad. Al mismo tiempo, llegaba a un conjunto más amplio de oyentes y otros interlocutores

48. CARUSO, Laura, Los trabajadores maritimos..., Op. Cit., capítulo 2.

49. El Marino No 4, 05/1938, p. 13, “Conferencias radiales", 9० Audición. buscados y deseados, como el Estado, sus instituciones y funcionarios.

Señores legisladores, señores gobernantes, en vuestras manos está la solución de nuestras dificultades; amigos oyentes, pedimos vuestro apoyo para nuestra justa causa, camaradas marítimos, cerrad filas en torno a la Unión Obrera Marítima $\mathrm{y}$ a las sociedades de Oficiales serias $\mathrm{y}$ responsables. ${ }^{50}$

De esta manera, el mensaje enunciado hacia la comunidad marítima y hacia las autoridades empresariales y estatales contribuía a conformar y reafirmar la idea de una comunidad carente de conflictos, producidos únicamente por agentes externos a la misma. En este mundo compartido existía un interés común realizable a través de la cooperación y conciliación, encarnado en las aspiraciones del gremio, interés común que para la UOM incluía el interés por el desarrollo de una marina mercante próspera y sin trabas. Este objetivo mejoraría las condiciones laborales, a la vez que

desde el punto de vista del interés de la Nación, aumenta el poder defensivo de esta sirve para hacerla conocer más... es obra altamente patriótica el mejoramiento de nuestras precarias condiciones de vida y obra altamente beneficiosa para el país la solución por parte de los poderes públicos de los inconvenientes que impiden el desarrollo normal de la marina mercante. ${ }^{51}$

Dicho reclamo sindical era entendido y justificado como una lucha por una "causa

50. El Marino No 3, 04/1938, p. 6, "Nuestras audiciones radiales", 40 Audición.

51.El Marino No 1, p. 3, "Las Conferencias Radiales de la Unión Obrera Marítima”. 
noble y justa, en beneficio de decenas de millares de argentinos y del progreso y defensa de la Nación misma". El tono nacionalista y patriótico, característico de esa década y ciertamente no privativo de esta organización gremial, pone de manifiesto la clave nacional autolegitimadora en la que esta enunciado, que posicionaba a la UOM más cerca de las autoridades patronales y gubernamentales.

Otro tópico en debate de las conferencias fueron los métodos empleados para hacer política gremial, con relación a la huelga y la acción directa, considerados violentos. Como se ha dicho, la UOM, en acuerdo con el Ministro de Obras Públicas, sostenía una firme oposición al uso de la violencia como medio para resolver las diferencias entre el capital y el trabajo, y que los diferenciaba de otros gremios:

Me dirijo a los camaradas marítimos. Los malos pastores con que desgraciadamente contó nuestro gremio... malos dirigentes que por no ser marítimos, mal podían interpretar nuestras necesidades, que, por haber convertido en profesión sus actividades de agitadores... Sólo los marítimos de verdad, que no responden a sectas o grupos interesados en producir entredichos entre armadores y tripulantes a fin de que les sirvan de campo de experimentación para sus elucubraciones o de base para satisfacer apetitos menos confesables, sólo los marítimos auténticos... son los que aspiran sinceramente a que la ley... sea pronto una bella realidad. ${ }^{52}$

En contraste con los métodos de protesta

52. La cita hace referencia al proyecto de Ley de Jubilaciones al que la FOM se opuso sistemáticamente. El Marino No 3, 04/1938, p. 5, "Nuestras audiciones radiales". 3० Audición. o acciones directa de la FOM, considerados contraproducentes en función de la experiencia previa, la UOM defendía el uso pleno de los espacios institucionales, la petición a los autoridades, la participación ciudadana, la protección gubernamental y la paz social con la patronal, consolidando un fuerte discurso de armonía de clases que informó su práctica gremial:

debemos echar mano de todos los recursos lícitos de que disponemos. Como electores, procurando interesar por nuestras aspiraciones a los hombres que nos piden nuestro voto. Como marítimos, apoyando las gestiones que nuevamente han de efectuar las instituciones ya mencionadas (UOM y Centro de Oficiales de la Marina Mercante), ante los poderes públicos. ${ }^{53}$

Entre otros, el voto era el recurso más lícito y legítimo, apelando al trabajador como ciudadano y elector, lo cual, en plena década de fraude, abstención electoral y exclusión política, parece al menos problemático. La defensa del uso pleno de los espacios institucionales para peticionar a las autoridades fue extendida $y$ plena, pensando al Estado como garante, y en cierta medida sustento, de la actividad gremial.

\section{Notas finales}

En el desarrollo masivo de la radio como fenómeno cultural y político el movimiento obrero no estuvo ausente. Como se ha analizado, algunas de sus organizaciones particulares y significativas, por su actividad y trayectoria, como el gremio marítimo, accedieron al espacio radial y se apropiaron

53. El Marino No 4, 05/1938, p. 13, "Conferencias radiales". 90 Audición. 
de éste nuevo medio para su construcción sindical. La UOM, un gremio menor por su peso relativo entre los trabajadores del sector, fue uno de los más visibles y de mayor vinculación con los poderes públicos. Logró poner al aire entre 1937 y 1938 una serie de conferencias aquí estudiadas, en las que desplegó una intensa actividad de propaganda de sus posiciones, demandas e intereses.

En principio, cabe señalar que en nuestro país la política en general, y la política gremial en particular, tardó en llegar al espacio radial. La experiencia analizada fue un intento interesante e ilustrativo, cuasi moderno, impulsado por la UOM. También interesa aquí mostrar, en este recorrido por los argumentos y el contenido de estas conferencias, que tales emisiones constituyeron un elemento más del juego político y la estrategia gremial desplegada por el sindicato, en particular en la búsqueda por vincularse al Estado, en dotar de legitimidad a sus reclamos y posiciones, y en la consolidación de su lugar frente a otros gremios con los que disputaba la representación sindical marítima. En tanto medio para difundir y hacer conocer sus necesidades y argumentos, tanto a quienes apuntaba a representar como a quienes reclamaba su protección, la UOM consideró fundamental realizar una intensa labor de propaganda político-sindical a través del éter, dando cuenta así de la importancia social y cultural que este medio había adquirido para fines de la década de 1930. Este estudio de caso permite mostrar cómo a través del espacio radial también se intentó desarrollar su política gremial, dar visibilidad a una organización ante los trabajadores del sector, las autoridades estatales y la comunidad obrera marítima en pleno, a la vez que apuntó a mejorar su posición frente a los gremios competidores. La radio constituyó así un nuevo medio de construcción sindical, acotado y tardío, sumándose a un repertorio vasto y variado, al que dio renovada fuerza.

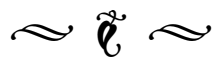

Recibido: 27/02/2014 Aceptado: 11/07/2014 Publicado: 31/07/2015 